\title{
Correction to: Oscillations and Pattern Formation in a Slow-Fast Prey-Predator System
}

\section{Pranali Roy Chowdhury ${ }^{1} \cdot$ Sergei Petrovskii ${ }^{2,3} \cdot$ Malay Banerjee $^{1}$}

Published online: 28 October 2021

(c) Society for Mathematical Biology 2021

\section{Correction to:}

\section{Bulletin of Mathematical Biology (2021) 83:110 https://doi.org/10.1007/s11538-021-00941-0}

The original version of the article, unfortunately, contained a few misprints. The notation of $\lambda_{H}$ was shown incorrectly in a few places in the published version. It has been corrected in this correction.

1. The Theorem 1 should be read as follows:

Theorem 1 Let $(U, V)=(0,0)$ be the canard point of the transformed system (12) at $\lambda=0$ such that $(0,0)$ is a folded singularity and $G(0,0,0)=0$. Then, for sufficiently small $\varepsilon$ there exist a singular Hopf bifurcation curve $\lambda=\lambda_{H}(\sqrt{\varepsilon})$ such that the equilibrium point $P$ of the system (12) is stable for $\lambda>\lambda_{H}(\sqrt{\varepsilon})$ and

$$
\lambda_{H}(\sqrt{\varepsilon})=-\frac{b_{3}\left(a_{1}+a_{5}\right)}{2 b_{2} b_{4}} \varepsilon+O\left(\varepsilon^{\frac{3}{2}}\right) .
$$

2. In Eq. (18), the expression on the left hand side $o_{H}(\sqrt{\varepsilon})$ should be read as $\delta_{H}(\sqrt{\varepsilon})$.

The original article can be found online at https://doi.org/10.1007/s11538-021-00941-0.

$凶 \quad$ Malay Banerjee

malayb@iitk.ac.in

Pranali Roy Chowdhury

pranali@iitk.ac.in

Sergei Petrovskii

sp237@leicester.ac.uk

1 Department of Mathematics and Statistics, Indian Institute of Technology Kanpur, Kanpur, Uttar Pradesh 208016, India

2 School of Computing and Mathematical Sciences, University of Leicester, Leicester LE1 7RH, UK

3 Peoples Friendship University of Russia (RUDN University), 6 Miklukho-Maklaya St, Moscow, Russian Federation 117198 
3. In the Appendix B, after Eq. (44), the expressions $\geq_{H}(\sqrt{\varepsilon})$ should be read as $\lambda_{H}(\sqrt{\varepsilon})$ at three places of occurrence.

The original article has been corrected.

Publisher's Note Springer Nature remains neutral with regard to jurisdictional claims in published maps and institutional affiliations. 